(c) $\quad A_{2}=8 \sum q^{n}\left(\sum(-1)^{d} \delta \cos 2 d y\right)-8 \sum q^{n}\left(\sum \delta\right)$,

(d) $A_{3}=1+8 \sum q^{n}\left(\sum(-1)^{t} \tau \cos 2 t y\right)-8 \sum q^{n}\left(\sum \delta\right)$,

(e) $\quad B_{0}=1 / 2+2 \sum q^{n}\left(\sum \delta\right)+8 \sum q^{n}\left(\sum(2 \tau-t) \cos 2 t y\right)$,

(f) $\quad B_{1}=-3 / 2+\csc ^{2} y+2 \sum q^{n}\left(\sum \delta\right)$

$$
+8 \sum q^{n}\left(\sum(2 \delta-d) \cos 2 d y\right) \text {, }
$$

(g) $\quad B_{2}=-3 / 2+\sec ^{2} y+2 \sum q^{n}\left(\sum \delta\right)$

$$
+8 \sum q^{n}\left(\sum(2 \delta-d)(-1)^{d} \cos 2 d y\right)
$$

(h) $\quad B_{3}=1 / 2+2 \sum q^{n}\left(\sum \delta\right)$

$$
+8 \sum q^{n}\left(\sum(-1)^{t}(2 \tau-t) \cos 2 t y\right) .
$$

UNIVERSITY OF ARKANSAS

\title{
A CONNECTEDNESS THEOREM IN ABSTRACT SETS*
}

BY W. M. WHYBURN

This note gives a variation of a theorem of Sierpinski and Saks. $\dagger$ The theorem is valid in spaces which have the BorelLebesgue property (Axiom I of Saks $\ddagger$ ) and which satisfy axioms (A), (B), (C), and (6) as given by Hausdorff. $\$$ We use the term connected for a closed set to mean that the set cannot be expressed as the sum of two mutually exclusive non-vacuous, closed sets.||

TheOREM. Let $F$ be a collection of closed sets at least one of which is compact. Let $F$ contain more than one element and let it be true that the sets of each finite sub-collection of $F$ have a nonvacuous, connected set in common when this sub-collection contains at least two elements of $F$. Under these hypotheses, there is a closed, non-vacuous, connected set common to all of the sets of collection $F$.

Proof. Let $F_{0}$ be a compact member of collection $F$ and let $K$ be the set of points common to all of the sets of collection $F$.

* Presented to the Society, December 1, 1934.

$\dagger$ See Saks, Fundamenta Mathematicae, vol. 2 (1921), pp. 1-3.

¥ Saks, ibid., p. 2.

\$ Mengenlehre, 1927, pp. 228-229.

|| The notion of limit point may be defined and this definition used to describe connectedness. We use domain and open set interchangeably. 
Saks* shows that $K$ is non-vacuous while the closure of $K$ is an immediate consequence of the closure of the sets of collection $F$ (since any point of the complement of $K$ has a neighborhood which belongs to the complement of some one of the sets of collection $F$ and hence belongs to the complement of $K$ ). It remains to show that $K$ is connected. Suppose $K=K_{1}+K_{2}$, where $K_{1}$ and $K_{2}$ are mutually exclusive, non-vacuous, closed sets. The collection $C$ composed of the complements of the sets of collection $F$ is a set of domains that covers $F_{0}-K$. By axiom (6), for each point $p$ of $K_{1}$ there exist mutually exclusive domains $G_{1 p}$ and $G_{2 p}$ such that $p \in G_{1 p}, K_{2} \cong G_{2 p}$. Let $\left[G_{1 p}\right]$ and $\left[G_{2 p}\right]$ be the collections of domains obtained in this manner for all points of $K_{1}$. The set $K_{1}$ is closed and compact and hence has the BorelLebesgue property. Let $G_{1}, \cdots, G_{n}$ be a finite sub-collection of $\left[G_{1 p}\right]$ which covers $K_{1}$ and let $H_{1}, \cdots, H_{n}$ be the corresponding members of $\left[G_{2 p}\right]$. If $H$ denotes the common part of $H_{1}, \cdots, H_{n}$, then $H$ is a domain that covers $K_{2}$ (this follows from a theorem stated by Hausdorff, loc. cit., page 229, line 4) while $G=G_{1}$ $+G_{2}+\cdots+G_{n}$ is a domain that covers $K_{1}$. Furthermore, $H$ and $G$ have no point in common since $G_{i}$ and $H_{i}$ are mutually exclusive sets. The collection $C$ together with $G$ and $H$ cover the closed and compact set $F_{0}$. The Borel-Lebesgue property yields a finite collection $C_{1}, C_{2}, \cdots, C_{m}, G, H$, of these sets that covers $F_{0}$ while the hypotheses of the theorem together with the method of construction of the covering sets force this collection to contain $G, H$, and at least one of the sets $C_{i}$. Let $F_{i}$ be the complement of $C_{i}$ and let $Q$ be the set common to $F_{0}, F_{1}, \cdots, F_{m}$. The set $Q$ contains $K_{1}$ and $K_{2}$ and is covered by $G$ and $H$ (since $Q$ belongs to $F_{0}$ and the complements of $C_{1}, C_{2}, \cdots, C_{m}$ ). Since $G$ and $H$ are mutually exclusive, it follows that $Q$ is not connected. This contradicts the hypothesis that any finite collection of two or more of the sets of $F$ has a connected set in common and yields the theorem.

The University of California at Los Angeles

* Loc. cit., p. 2. 